\title{
HTLV-1 bZIP factor gene: Its roles in HTLV-1 pathogenesis.
}

$\operatorname{AUTHOR}(S)$ :

Matsuoka, Masao

CITATION:

Matsuoka, Masao. HTLV-1 bZIP factor gene: Its roles in HTLV-1

pathogenesis.. Molecular aspects of medicine 2010, 31(5): 359-366

ISSUE DATE:

2010-10

URL:

http://hdl.handle.net/2433/131803

\section{RIGHT:}

(C) 2010 Elsevier Ltd; この論文は出版社版でありません。引用の際には 出版社版をご確認ご利用ください。; This is not the published version. Please cite only the published version. 


\title{
HTLV-1 bZIP factor gene: its roles in HTLV-1 pathogenesis
}

\author{
Masao Matsuoka
}

Institute for Virus research, Kyoto University

53 Shogoin Kawahara-cho, Sakyo-ku, Kyoto 606-8507, Japan 


\section{Abstract}

The HTLV-1 bZIP factor (HBZ) gene is transcribed as an antisense transcript of HTLV-1 from the 3' long terminal repeat (LTR). Recent studies showed that the HBZ gene was expressed in all ATL cases, suggesting its critical role in leukemogenesis. In addition, only the HBZ gene sequence remains intact, unaffected by nonsense mutations and deletion. HBZ mRNA promotes proliferation of adult T-cell leukemia (ATL) cells. The HBZ protein has three domains: activation, central and bZIP domains. HBZ interacts with a variety of cellular factors, and modulates not only cellular functions, but also viral gene transcription from 5'LTR. The complex functions of HBZ modulate T-cells, and promote their proliferation, which is likely indispensable for leukemogenesis by HTLV-1. 


\section{Introduction}

Human T-cell leukemia virus type 1 (HTLV-1) was first reported in 1980 (Poiesz et al., 1980). Thereafter, its causative association with adult T-cell leukemia (ATL) was clarified (Hinuma et al., 1981). The whole sequence was reported by Yoshida's group (Seiki et al., 1983), who revealed the unique region between env and 3' long terminal repeat (LTR), designated the $\mathrm{pX}$ region. The $\mathrm{pX}$ region encodes regulatory genes, $\operatorname{tax}$ and rex, in addition to accessory genes, $p 12, p 13$, and $p 30$. At that time, researchers of HTLV-1 noticed an open reading frame in the minus strand. The presence of an anti-sense transcript of HTLV-1 was first detected by Northern blot in HTLV-1-infected cell lines (Larocca et al., 1989). However, the function and exact structure of this gene product remained unknown. This is because researchers naturally focused on Tax, a fascinating viral protein with pleiotropic actions, and the concept of antisense transcription was not so common at that time. In 2002, a viral protein that binds to CREB-2 was found by yeast two-hybrid screening, and named HTLV-1 bZIP factor (HBZ). HBZ was first found to inhibit viral gene transcription of the sense strand (Gaudray et al., 2002). In a separate study, we analyzed HTLV-1 provirus in ATL cells, and reported that Tax expression was frequently disrupted by several mechanisms (Matsuoka and Jeang, 2007). However, the $H B Z$ gene sequence, as well as the 3'LTR that is its promoter, remained intact (Fan et al., 2010; Yoshida et al., 2008). In addition, the spliced form of the $H B Z$ gene transcript was identified and was expressed in all ATL cases. Importantly, $H B Z$ gene expression promoted proliferation of ATL cells (Satou et al., 2006). Thus, HBZ is now recognized as a critical gene for ATL cells. In this review, 
recent findings on HBZ are summarized, and its roles in HTLV-1 infectivity and oncogenesis discussed.

1. Structure and transcription of the $H B Z$ gene

Transcription start sites of the $H B Z$ gene were identified by 5' RACE (Cavanagh et al., 2006; Murata et al., 2006; Satou et al., 2006). These studies revealed different transcripts of the $H B Z$ gene: a spliced form (sHBZ) and an unspliced form (usHBZ)(Figure 1). The first exon of the $s H B Z$ gene transcript is present in $\mathrm{U} 3$ and $\mathrm{R}$ regions of the 3'LTR. The difference between sHBZ and usHBZ is only a few amino acids, as shown in Figure 1B. Transcription start sites of the spliced $H B Z$ gene were scattered in the U5 and R regions of the 3'LTR, which is consistent with the finding that the predicted promoter was TATA-less (Yoshida et al., 2008). Analyses of transcription factor binding sites of the promoter region showed that three Sp1 binding sites were critical for transcription of the $H B Z$ gene while the third Sp1 site was most important. Binding of $\mathrm{Sp} 1$ to this site was proved by chromatin immunoprecipitation. It has been reported that $\mathrm{Sp} 1$ is critical for many TATA-less promoters (Boam et al., 1995; Liu and Cowell, 2000). Since Sp1 is a well-known regulator of housekeeping genes, transcription of the $\mathrm{s} H B Z$ gene may be relatively constant. Taken together, $\mathrm{Sp} 1$ is critical for transcription of the $H B Z$ gene. As described below, the $H B Z$ gene transcript is better correlated with provirus load than the tax gene transcript (Saito et al., 2009), indicating that the $H B Z$ gene is constantly expressed in HTLV-1 infected cells. Thus, transcription from the minus strand contrasts with that from the 5'LTR on the plus 
strand, which is highly inducible by Tax. Viral genes other than the $H B Z$ gene are transcribed by the 5'LTR and HBZ suppresses transcription from the 5'LTR. This is an ingenious mechanism to control expression of viral proteins. Tax can activate transcription of the HBZ gene through a Tax responsive element (TRE) (Landry et al., 2009; Yoshida et al., 2008). However, its enhancement is not so significant, and Tax expression is usually controlled in vivo. Therefore, the significance of Tax mediated upregulation of the $H B Z$ gene transcription remains to be determined.

Quantitative analyses of the $H B Z$ gene transcripts were reported by two groups (Saito et al., 2009; Usui et al., 2008). Transcripts of the spliced form of the $H B Z$ gene were four- fold more abundant than those of usHBZ (Usui et al., 2008). This observation correlates with the finding that the promoter activity of the s $H B Z$ gene was much higher than that of the us $H B Z$ gene (Yoshida et al., 2008). Relative expression level of the $H B Z$ gene that was adjusted by provirus load was almost equivalent among HTLV-1 carriers, HAM/TSP patients and ATL patients (Saito et al., 2009). A previous study reported that HTLV-1 provirus load was correlated with the expression level of the tax gene (Yamano et al., 2002). However, the $H B Z$ gene transcript was more closely correlated with provirus load than was the level of the tax gene transcripts (Saito et al., 2009). Kinetic study of the sHBZ gene transcripts in rabbits shows that $\mathrm{s} H B Z$ gene transcription was detected one week post-infection and increased and stabilized, while other viral genes were at or below the limit of detection (Li et al., 2009). This finding supports a correlation between sHBZ gene expression, provirus load, and survival of HTLV-1 infected cells. 
It is noteworthy that the $H B Z$ mRNA was well correlated with disease severity in HAM/TSP patients. In addition, neopterin in the cerebrospinal cord fluid, which reflects cellular immune responses, was positively correlated with the level of HBZ mRNA, but not with tax mRNA. Taken together, these results show that the $H B Z$ gene expression is closely linked with disease severity of HAM/TSP, suggesting that the $H B Z$ gene expression plays critical roles in proliferation of HTLV-1 infected cells and pathogenesis.

2. The $H B Z$ gene in ATL cells

HTLV-1 provirus is the only evidence of HTLV-1 infection in ATL cells, and analysis of provirus is expected to provide clues on leukemogenesis, and on which viral gene is critical for ATL cells or preferential integration sites of HTLV-1 provirus in leukemic cells. It has been thought that Tax is critical for leukemogenesis due to the abundance of reports on its potent effect on cell proliferation, genetic instability, and dysregulation of the cell cycle (Grassmann et al., 2005). Tax expression in transgenic animals induces cancers depending on the promoter used (Lairmore et al., 2005). For example, transgenic mice that expressed Tax under control of the human granzyme B gene promoter developed natural killer cell leukemia while salivary and mammary adenomas were found in the transgenic mice expressing Tax under the CD3-epsilon promoter-enhancer. In vitro expression of Tax could immortalize human T-lymphocytes although Tax expression could not transform T-lymphoctes (Akagi et al., 1995). However, Tax expression was not detected in about $60 \%$ of freshly isolated samples 
from ATL cases (Takeda et al., 2004), indicating that Tax expression is not always necessary for ATL. Detailed analyses of HTLV-1 provirus showed three mechanisms to disrupt Tax expression in ATL cells (Figure 2). Firstly, the 5'LTR, which is a promoter/enhancer of viral gene transcription of the plus strand, was deleted (Miyazaki et al., 2007; Tamiya et al., 1996). Secondly, CpG sites of the 5'LTR were hypermethylated, which lead to silencing of plus strand viral gene transcription (Koiwa et al., 2002; Takeda et al., 2004; Taniguchi et al., 2005). Finally, genetic changes (nonsense mutations, deletions, and insertions) were found in the tax gene itself (Furukawa et al., 2001; Takeda et al., 2004). These findings suggest that Tax expression is not necessary in the late steps of the leukemogenic process although its expression is required at an early stage of ATL or in the carrier state. However, an analysis of defective provirus without a 5'LTR showed that 8 of 12 cases retained 6 bp short repeats at both ends of the LTR, while 4 cases lacked these sequences. Since these short repeats are generated during integration, the defective provirus with the 6-bp short repeat is formed before integration. Importantly, defective proviruses in two of eight cases lacked the second exon of the tax gene (Miyazaki et al., 2007), indicating that Tax was not expressed in these cases prior to development of leukemia. Nonsense mutations in the tax gene by APOBEC3G were found in 7 of 60 ATL cases (Fan et al., 2010). Similar nonsense mutations of the $\operatorname{tax}$ gene were also found in the carrier state. Thus, Tax expression is not necessary at least for these cases. Conversely, nonsense mutations were not found in the HBZ gene in both ATL cases and HTLV-1 carriers, suggesting that HBZ expression is indispensable for proliferation and survival of ATL cells and 
HTLV-1 infected cells.

As described, three mechanisms prevent Tax expression in ATL cells. However, the 3'LTR remained unmethylated and intact in all of the ATL cases, indicating that the 3'LTR was critical for ATL cells. Since viral genes other than the $H B Z$ gene are transcribed from the 5'LTR that is frequently silenced or lost, we expected $H B Z$ to be the only viral gene that is consistently expressed in ATL cells. In fact, the $H B Z$ gene was transcribed in all ATL cases while transcripts of the tax gene were detected in only a limited number of cases (Satou et al., 2006). Suppressing expression of the $H B Z$ gene inhibited proliferation of ATL cells (Satou 2006), indicating that HBZ has growth-promoting activity in ATL.

\section{Property and function of HBZ protein}

The spliced transcript of HBZ is translated into a polypeptide of 206 amino acids, while the protein product of unspliced HBZ is a polypeptide of 209 amino acids. HBZ has three domains; the activation, central and bZIP domains (Figure 3). HBZ protein is localized in the nucleus with a speckled pattern. Three regions are associated with nuclear localization: two regions rich in basic amino acids and a DNA binding domain (Figure 3)(Hivin et al., 2005). In addition, the integrity of the HBZ amino acid sequence is necessary for the speckled distribution in the nucleus. HBZ is localized in heterochromatin, consistent with its association with transcriptional inhibition (Hivin et al., 2005). Furthermore, HBZ has been shown to sequester JunB to nuclear bodies, thus suppressing JunB-dependent transcriptional activity (Hivin et al., 2007). 
HBZ is not necessary for in vitro transformation of T-cells although mutation of the HBZ gene resulted in decreased proliferation of infected cells in vivo (Arnold et al., 2006), implicating HBZ in infectivity and viral persistence.

1) Suppression of Tax mediated viral gene transcription

HBZ was first found as a viral protein that binds to CREB2 (ATF-4) (Gaudray et al., 2002). Through bZIP domain interaction, HBZ abolished the ability of CREB-2 to bind to the Tax responsive element (TxRE) in the HTLV-1 LTR, resulting in the suppression of transcription from the 5'LTR by Tax (Figure 4). Moreover, HBZ interacts with cellular coactivators $\mathrm{CBP} / \mathrm{p} 300$, via LXXLL-like motifs in its $\mathrm{N}$ terminal region, leading to suppression of viral transcription by inhibiting the recruitment of $\mathrm{CBP} / \mathrm{p} 300$ to the HTLV-1 promoter (Clerc et al., 2008). So far, there is no report that HBZ has the capacity to associate with DNA directly. It is apparent that HBZ exerts its suppressive effect on HTLV-1 transcription mainly by interacting with cellular proteins on the HTLV-1 promoter.

2) Interaction of HBZ with cellular factors with bZIP domains The identification of other cellular factors that interact with HBZ was undertaken on an individual basis after analyses of the factors suspected to be involved in the signal pathway of ATL. HBZ, via its bZIP domain, reportedly forms heterodimers with several AP-1 transcriptional family members, such as c-Jun, JunB, and JunD, but not c-Fos, and modulates their activity (Figure 4)(Basbous et al., 2003; Thebault et al., 2004). Analysis 
revealed that HBZ decreases the DNA binding capability of c-Jun and JunB, as is the case for ATF-4 and CREB. JunB was sequestrated into nuclear bodies through interaction with HBZ (Hivin et al., 2007). For c-Jun, HBZ enhances degradation of c-Jun, as described later, and inhibits its DNA binding (Figure 4). Interactions between HBZ and other bZIP factors, such as ATF1 and ATF2, have also been shown (Isono et al., 2008; Lemasson et al., 2007). Recent study using coiled-coil arrays revealed binding of factors containing bZIP domains with HBZ (Reinke et al., 2010). The function of such associations remains to be elucidated.

3) Activation of transcription by interacting with JunD

HBZ suppresses transcription mediated by c-Jun and JunB. However, HBZ can also activate transcription mediated by JunD, another member of the AP-1 family. HBZ forms heterodimers with JunD via its bZIP domain, and the activation domain of HBZ is necessary for this activation (Thebault et al., 2004). In addition to this activity, HBZ JunD heterodimers interact with $\mathrm{Sp} 1$ and activate transcription of the human telomerase catalytic subunit (hTERT) (Figure 4) (Kuhlmann et al., 2007).

4) Inhibition of the classical NF-אB pathway

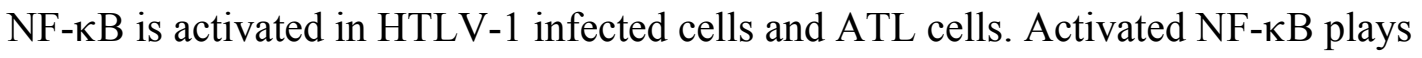
important roles in the proliferation of ATL cells and inhibition of apoptosis (Sun and Yamaoka, 2005). Tax can activate both the classical and alternative pathways of NF- $\kappa B$. Tax binds to $\mathrm{IkB}$, resulting in dissociation of the $\mathrm{I} \kappa \mathrm{B} / \mathrm{NF}-\kappa \mathrm{B}$ complex and promoting 
nuclear translocation of NF-KB (Suzuki et al., 1995). A further mechanism is Tax enhancing I $\kappa \mathrm{B} \alpha$ degradation by interacting with $\operatorname{IKK} \gamma$, leading to subsequent activation

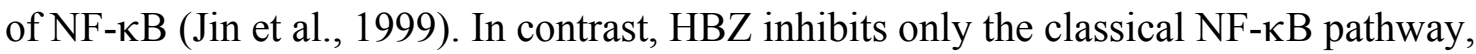
doing so by two mechanisms (Figure 4). One mechanism is that HBZ inhibits DNA binding of p65. Another is that HBZ increases the expression of PDLIM2, the E3 ubiquitin ligase of p65, leading to increased ubiquitination and enhanced degradation of p65 (Zhao et al., 2009).

Many viruses have developed strategies to manipulate NF- $\kappa B$ signaling through the use of multifunctional viral proteins. HIV-1 Nef induces the expression of the NF- $\kappa \mathrm{B}$ inhibitor IкB $\alpha$, to suppress this pathway (Qiao et al., 2006). In Epstein-Barr virus, the LMP-1 viral protein activates the NF-אB pathway by recruiting cellular adaptor proteins, TNF receptor associated factor families and TNF receptor-associated death domain, to its C-terminal domain. Like HBZ, the EBV bZIP protein, BZLF1, suppresses the

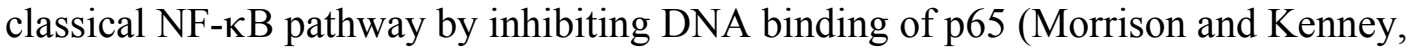
2004). Similar suppression of NF- $\kappa B$ has been reported for other viruses, including African swine fever virus, and human herpesvirus-8 (HHV-8). In the case of HHV-8, vFLIP activates both the classical and alternative pathways while viral interferon

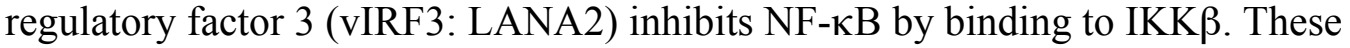
findings show that NF- $\mathrm{KB}$ suppressive activities are common among different viruses, suggesting that these suppressive activities are important for viral infection. A virus might escape from the host immune system by suppressing the classical NF- $\kappa B$ pathway. 
4. The proteasome and HBZ

One mechanism by which HBZ inhibits transcriptional activation by c-Jun and JunB (Basbous et al., 2003) is by forming heterodimers, and thereby inhibiting their DNA binding. HBZ further inhibits c-Jun by promoting the degradation of its protein product (Figure 4) (Matsumoto et al., 2005). Since it can be blocked by a proteasome inhibitor, this effect relies on a protesome-mediated pathway. Degradation of c-Jun does not, however, depend on ubiquitination (Isono et al., 2008). usHBZ protein directly interacts with both the $26 \mathrm{~S}$ proteasome and c-Jun, which results in the delivery of c-Jun to the proteasome. Thus, HBZ suppresses c-Jun by three mechanisms; 1) inhibition of DNA binding, 2) ubiquitin-independent degradation of c-Jun, and 3) sequestration of c-Jun in nuclear bodies.

It has been reported that degradation of c-Jun by sHBZ is much weaker than that by usHBZ (Isono et al., 2008). However, inhibition of AP-1 mediated transcription by sHBZ was much stronger than that of usHBZ (Yoshida et al., 2008). For the sHBZ protein, the inhibition of DNA binding by c-Jun or sequestration in nuclear bodies might be the predominant mechanism of transcriptional suppression. Further, the expression level of sHBZ RNA is much higher than that of usHBZ RNA, and the half-life of sHBZ protein is much longer than that of usHBZ. Only one HBZ protein was detected in ATL cell lines (Arnold et al., 2008). Taken together, sHBZ expression is much higher than that of usHBZ, and only sHBZ RNA has growth-promoting activity as described later, indicating that sHBZ is more important than usHBZ for HTLV-1 
infected cells and ATL cells.

\section{Function of the HBZ gene as RNA}

Suppressed expression of the HBZ gene by shRNA leads to decreased proliferation of ATL cell lines (Arnold et al., 2008; Satou et al., 2006). Expression of HBZ in transgenic mice increases the number of T-cells (Satou et al., 2006), and tumor formation and infiltration of ATL cells is decreased by suppressed HBZ expression (Arnold et al., 2008). Thus, HBZ expression is associated with proliferation of ATL cells in vivo and in vitro. Mutation analyses of the $H B Z$ gene showed that $H B Z$ RNA, rather than $H B Z$ protein, has a growth promoting effect on T-cells (Figure 4) (Satou et al., 2006). The coding sequence of the $H B Z$ gene was replaced with silent mutations, which could produce the same protein while the RNA structure was completely altered. This mutant did not have a growth-promoting activity. Analysis of the transcription profile using DNAchip demonstrated that expression of the HBZ gene upregulates E2F1 gene transcription. Only sHBZ RNA, not usHBZ RNA, promotes proliferation of T-cells, indicating that the first exon of the sHBZ transcript is critical for this activity (Yoshida et al., 2008). This exon overlaps with the Rex responsive element (RxRE) in the $\mathrm{R}$ region of 3'LTR. The RxRE region forms a stem-loop structure that binds to Rex. Rex promotes the export of viral RNA with a RxRE region, Thus, sHBZ RNA functions to promote proliferation of T-cells by a region containing the first exon, which can form a strong stem-loop structure. Further details of how sHBZ RNA promotes proliferation remain to be elucidated. 


\section{Antisense transcript of HTLV-2}

HTLV-2 is similar to HTLV-1: both retroviruses target T-lymphocytes, and induce their proliferation. However, HTLV-2 tends to infect CD8+ T-cells instead of CD4+ T-cells (Kwaan et al., 2006), and induces cancer in humans only in very rare cases. Following the discovery of HBZ, an antisense transcript was identified in HTLV-2. Its product was named antisense protein of HTLV-2 (APH-2)(Halin et al., 2009). APH-2 mRNA is transcribed from the 3'LTR, and spliced and polyadenylated like the sHBZ gene. Although APH-2 does not have a basic leucine zipper domain, it interacts with CREB and suppresses Tax2 mediated transcription. Thus, APH-2 has similar structure and functions to sHBZ. However, it remains to be elucidated whether APH-2 induces proliferation of infected lymphocytes in vivo.

\section{HBZ gene transcript and diseases}

The $H B Z$ gene is the only viral gene that is consistently expressed in HTLV-1 infected cells and ATL cells, indicating that HBZ plays a critical role in both infected cells and leukemic cells. For ATL cells, HBZ RNA has growth promoting activity. ATL cells frequently express Foxp3, a master factor of regulatory T-cells. HBZ expression is associated with a phenotype of regulatory T-cells (our unpublished data). Thus, HBZ not only promotes proliferation of ATL cells, but also modulates the phenotype of HTLV-1 infected cells.

The expression level of $H B Z$ transcripts is closely correlated with provirus load and the 
disease severity of HAM/TSP (Saito et al., 2009). Increased infected cells are also implicated in the pathogenesis of HAM/TSP. There are two scenarios for the role of HBZ: 1) HBZ is important for the survival of HTLV-1 infected cells in vivo, 2) HBZ expression confers infiltrative phenotypes on infected cells, and causes dysregulation of cytokine production. The role of HBZ gene in HAM/TSP should be studied in the future.

\section{Perspectives}

A limited number of viral genes are implicated in virus-induced oncogenesis. Both E6 and E7 are critical for human papilloma virus (HPV)-induced cervical cancer. In hepatitis B virus-induced hepatoma, HBx protein is a critical viral factor for oncogenesis. The pleiotropic functions of Tax are thought to play important roles in HTLV-1, however, this does not explain the frequent loss of Tax expression from ATL. It is hypothesized that Tax expression is necessary in the early stages of leukemia, but not required in late stages. However, HBZ is expressed in all ATL cases, and promotes proliferation of ATL cells. This indicates that HBZ is a critical viral gene in oncogenesis by HTLV-1. A few ATL cases had HTLV-1 provirus that could not produce Tax before its integration (Miyazaki et al., 2007; Tamiya et al., 1996). In addition, nonsense mutations of viral genes except the $H B Z$ gene are generated by APOBEC3G before integration of provirus (Fan et al., 2010). These findings suggest that the $H B Z$ gene is responsible for leukemogenesis by HTLV-1. Like other viral proteins of oncogenic viruses, recent studies show the multiple functions of HBZ. 
Further studies of HBZ gene will shed light on leukemogenesis by HTLV-1. Further, HBZ might be a novel target for prevention of, and therapies for, HTLV-1-associated diseases.

\section{References}

Akagi, T., Ono, H., and Shimotohno, K. (1995). Characterization of T cells immortalized by Tax1 of human T-cell leukemia virus type 1. Blood 86, 4243-4249. Arnold, J., Yamamoto, B., Li, M., Phipps, A.J., Younis, I., Lairmore, M.D., and Green, P.L. (2006). Enhancement of infectivity and persistence in vivo by HBZ, a natural antisense coded protein of HTLV-1. Blood 107, 3976-3982.

Arnold, J., Zimmerman, B., Li, M., Lairmore, M.D., and Green, P.L. (2008). Human T-cell leukemia virus type-1 antisense-encoded gene, Hbz, promotes T-lymphocyte proliferation. Blood 112, 3788-3797.

Basbous, J., Arpin, C., Gaudray, G., Piechaczyk, M., Devaux, C., and Mesnard, J.M. (2003). The HBZ factor of human T-cell leukemia virus type I dimerizes with transcription factors JunB and c-Jun and modulates their transcriptional activity. The Journal of biological chemistry 278, 43620-43627.

Boam, D.S., Davidson, I., and Chambon, P. (1995). A TATA-less promoter containing binding sites for ubiquitous transcription factors mediates cell type-specific regulation of the gene for transcription enhancer factor-1 (TEF-1). The Journal of biological chemistry 270, 19487-19494.

Cavanagh, M.H., Landry, S., Audet, B., Arpin-Andre, C., Hivin, P., Pare, M.E., Thete, J., Wattel, E., Marriott, S.J., Mesnard, J.M., et al. (2006). HTLV-I antisense transcripts initiating in the 3' LTR are alternatively spliced and polyadenylated. Retrovirology 3, 15.

Clerc, I., Polakowski, N., Andre-Arpin, C., Cook, P., Barbeau, B., Mesnard, J.M., and Lemasson, I. (2008). An interaction between the human T cell leukemia virus type 1 basic leucine zipper factor (HBZ) and the KIX domain of p300/CBP contributes to the down-regulation of tax-dependent viral transcription by HBZ. The Journal of biological 
chemistry 283, 23903-23913.

Fan, J., Ma, G., Nosaka, K., Tanabe, J., Satou, Y., Koito, A., Wain-Hobson, S., Vartanian, J.P., and Matsuoka, M. (2010). APOBEC3G Generates Nonsense Mutations in HTLV-1 Proviral Genomes In Vivo. Journal of virology.

Furukawa, Y., Kubota, R., Tara, M., Izumo, S., and Osame, M. (2001). Existence of escape mutant in HTLV-I tax during the development of adult T-cell leukemia. Blood 97, 987-993.

Gaudray, G., Gachon, F., Basbous, J., Biard-Piechaczyk, M., Devaux, C., and Mesnard, J.M. (2002). The complementary strand of the human T-cell leukemia virus type 1 RNA genome encodes a bZIP transcription factor that down-regulates viral transcription. Journal of virology $76,12813-12822$.

Grassmann, R., Aboud, M., and Jeang, K.T. (2005). Molecular mechanisms of cellular transformation by HTLV-1 Tax. Oncogene 24, 5976-5985.

Halin, M., Douceron, E., Clerc, I., Journo, C., Ko, N.L., Landry, S., Murphy, E.L., Gessain, A., Lemasson, I., Mesnard, J.M., et al. (2009). Human T-cell leukemia virus type 2 produces a spliced antisense transcript encoding a protein that lacks a classic bZIP domain but still inhibits Tax2-mediated transcription. Blood 114, 2427-2438. Hinuma, Y., Nagata, K., Hanaoka, M., Nakai, M., Matsumoto, T., Kinoshita, K.I., Shirakawa, S., and Miyoshi, I. (1981). Adult T-cell leukemia: antigen in an ATL cell line and detection of antibodies to the antigen in human sera. Proc Natl Acad Sci U S A $78,6476-6480$.

Hivin, P., Basbous, J., Raymond, F., Henaff, D., Arpin-Andre, C., Robert-Hebmann, V., Barbeau, B., and Mesnard, J.M. (2007). The HBZ-SP1 isoform of human T-cell leukemia virus type I represses JunB activity by sequestration into nuclear bodies. Retrovirology 4, 14.

Hivin, P., Frederic, M., Arpin-Andre, C., Basbous, J., Gay, B., Thebault, S., and Mesnard, J.M. (2005). Nuclear localization of HTLV-I bZIP factor (HBZ) is mediated by three distinct motifs. J Cell Sci 118, 1355-1362.

Isono, O., Ohshima, T., Saeki, Y., Matsumoto, J., Hijikata, M., Tanaka, K., and Shimotohno, K. (2008). Human T-cell leukemia virus type $1 \mathrm{HBZ}$ protein bypasses the targeting function of ubiquitination. The Journal of biological chemistry 283, 34273-34282.

Jin, D.Y., Giordano, V., Kibler, K.V., Nakano, H., and Jeang, K.T. (1999). Role of 
adapter function in oncoprotein-mediated activation of NF-kappaB. Human T-cell leukemia virus type I Tax interacts directly with IkappaB kinase gamma. The Journal of biological chemistry 274, 17402-17405.

Koiwa, T., Hamano-Usami, A., Ishida, T., Okayama, A., Yamaguchi, K., Kamihira, S., and Watanabe, T. (2002). 5'-long terminal repeat-selective CpG methylation of latent human T-cell leukemia virus type 1 provirus in vitro and in vivo. Journal of virology 76 , 9389-9397.

Kuhlmann, A.S., Villaudy, J., Gazzolo, L., Castellazzi, M., Mesnard, J.M., and Duc Dodon, M. (2007). HTLV-1 HBZ cooperates with JunD to enhance transcription of the human telomerase reverse transcriptase gene (hTERT). Retrovirology 4, 92.

Kwaan, N., Lee, T.H., Chafets, D.M., Nass, C., Newman, B., Smith, J., Garratty, G., and Murphy, E.L. (2006). Long-term variations in human T lymphotropic virus (HTLV)-I and HTLV-II proviral loads and association with clinical data. J Infect Dis 194, 1557-1564.

Lairmore, M.D., Silverman, L., and Ratner, L. (2005). Animal models for human T-lymphotropic virus type 1 (HTLV-1) infection and transformation. Oncogene 24, 6005-6015.

Landry, S., Halin, M., Vargas, A., Lemasson, I., Mesnard, J.M., and Barbeau, B. (2009). Upregulation of human T-cell leukemia virus type 1 antisense transcription by the viral tax protein. Journal of virology 83, 2048-2054.

Larocca, D., Chao, L.A., Seto, M.H., and Brunck, T.K. (1989). Human T-cell leukemia virus minus strand transcription in infected T-cells. Biochemical and biophysical research communications $163,1006-1013$.

Lemasson, I., Lewis, M.R., Polakowski, N., Hivin, P., Cavanagh, M.H., Thebault, S., Barbeau, B., Nyborg, J.K., and Mesnard, J.M. (2007). Human T-cell leukemia virus type 1 (HTLV-1) bZIP protein interacts with the cellular transcription factor CREB to inhibit HTLV-1 transcription. Journal of virology 81, 1543-1553.

Li, M., Kesic, M., Yin, H., Yu, L., and Green, P.L. (2009). Kinetic analysis of human T-cell leukemia virus type 1 gene expression in cell culture and infected animals. Journal of virology $83,3788-3797$.

Liu, S., and Cowell, J.K. (2000). Cloning and characterization of the TATA-less promoter from the human GFI1 proto-oncogene. Annals of human genetics 64, 83-86. Matsumoto, J., Ohshima, T., Isono, O., and Shimotohno, K. (2005). HTLV-1 HBZ 
suppresses AP-1 activity by impairing both the DNA-binding ability and the stability of c-Jun protein. Oncogene 24, 1001-1010.

Matsuoka, M., and Jeang, K.T. (2007). Human T-cell leukaemia virus type 1 (HTLV-1) infectivity and cellular transformation. Nature reviews 7, 270-280.

Miyazaki, M., Yasunaga, J., Taniguchi, Y., Tamiya, S., Nakahata, T., and Matsuoka, M. (2007). Preferential Selection of Human T-Cell Leukemia Virus Type 1 Provirus Lacking the 5' Long Terminal Repeat during Oncogenesis. Journal of virology 81, 5714-5723.

Morrison, T.E., and Kenney, S.C. (2004). BZLF1, an Epstein-Barr virus immediate-early protein, induces p65 nuclear translocation while inhibiting p65 transcriptional function. Virology 328, 219-232.

Murata, K., Hayashibara, T., Sugahara, K., Uemura, A., Yamaguchi, T., Harasawa, H., Hasegawa, H., Tsuruda, K., Okazaki, T., Koji, T., et al. (2006). A novel alternative splicing isoform of human T-cell leukemia virus type 1 bZIP factor (HBZ-SI) targets distinct subnuclear localization. Journal of virology 80, 2495-2505.

Poiesz, B.J., Ruscetti, F.W., Gazdar, A.F., Bunn, P.A., Minna, J.D., and Gallo, R.C. (1980). Detection and isolation of type $C$ retrovirus particles from fresh and cultured lymphocytes of a patient with cutaneous T-cell lymphoma. Proc Natl Acad Sci U S A 77, 7415-7419.

Qiao, X., He, B., Chiu, A., Knowles, D.M., Chadburn, A., and Cerutti, A. (2006). Human immunodeficiency virus 1 Nef suppresses CD40-dependent immunoglobulin class switching in bystander B cells. Nat Immunol 7, 302-310.

Reinke, A.W., Grigoryan, G., and Keating, A.E. (2010). Identification of bZIP interaction partners of viral proteins HBZ, MEQ, BZLF1, and K-bZIP using coiled-coil arrays. Biochemistry 49, 1985-1997.

Saito, M., Matsuzaki, T., Satou, Y., Yasunaga, J., Saito, K., Arimura, K., Matsuoka, M., and Ohara, Y. (2009). In vivo expression of the HBZ gene of HTLV-1 correlates with proviral load, inflammatory markers and disease severity in HTLV-1 associated myelopathy/tropical spastic paraparesis (HAM/TSP). Retrovirology 6, 19.

Satou, Y., Yasunaga, J., Yoshida, M., and Matsuoka, M. (2006). HTLV-I basic leucine zipper factor gene mRNA supports proliferation of adult $\mathrm{T}$ cell leukemia cells. Proc Natl Acad Sci U S A 103, 720-725.

Seiki, M., Hattori, S., Hirayama, Y., and Yoshida, M. (1983). Human adult T-cell 
leukemia virus: complete nucleotide sequence of the provirus genome integrated in leukemia cell DNA. Proc Natl Acad Sci U S A 80, 3618-3622.

Sun, S.C., and Yamaoka, S. (2005). Activation of NF-kappaB by HTLV-I and implications for cell transformation. Oncogene 24, 5952-5964.

Suzuki, T., Hirai, H., Murakami, T., and Yoshida, M. (1995). Tax protein of HTLV-1 destabilizes the complexes of NF-kappa B and I kappa B-alpha and induces nuclear translocation of NF-kappa B for transcriptional activation. Oncogene 10, 1199-1207. Takeda, S., Maeda, M., Morikawa, S., Taniguchi, Y., Yasunaga, J., Nosaka, K., Tanaka, Y., and Matsuoka, M. (2004). Genetic and epigenetic inactivation of tax gene in adult T-cell leukemia cells. Int J Cancer 109, 559-567.

Tamiya, S., Matsuoka, M., Etoh, K., Watanabe, T., Kamihira, S., Yamaguchi, K., and Takatsuki, K. (1996). Two types of defective human T-lymphotropic virus type I provirus in adult T-cell leukemia. Blood 88, 3065-3073.

Taniguchi, Y., Nosaka, K., Yasunaga, J., Maeda, M., Mueller, N., Okayama, A., and Matsuoka, M. (2005). Silencing of human T-cell leukemia virus type I gene transcription by epigenetic mechanisms. Retrovirology 2, 64 .

Thebault, S., Basbous, J., Hivin, P., Devaux, C., and Mesnard, J.M. (2004). HBZ interacts with JunD and stimulates its transcriptional activity. FEBS Lett 562, 165-170. Usui, T., Yanagihara, K., Tsukasaki, K., Murata, K., Hasegawa, H., Yamada, Y., and Kamihira, S. (2008). Characteristic expression of HTLV-1 basic zipper factor (HBZ) transcripts in HTLV-1 provirus-positive cells. Retrovirology 5, 34.

Yamano, Y., Nagai, M., Brennan, M., Mora, C.A., Soldan, S.S., Tomaru, U., Takenouchi, N., Izumo, S., Osame, M., and Jacobson, S. (2002). Correlation of human T-cell lymphotropic virus type 1 (HTLV-1) mRNA with proviral DNA load, virus-specific CD8(+) T cells, and disease severity in HTLV-1-associated myelopathy (HAM/TSP). Blood 99, 88-94.

Yoshida, M., Satou, Y., Yasunaga, J., Fujisawa, J., and Matsuoka, M. (2008).

Transcriptional control of spliced and unspliced human T-cell leukemia virus type 1 bZIP factor (HBZ) gene. J Virol 82, 9359-9368.

Zhao, T., Yasunaga, J., Satou, Y., Nakao, M., Takahashi, M., Fujii, M., and Matsuoka, M. (2009). Human T-cell leukemia virus type $1 \mathrm{bZIP}$ factor selectively suppresses the classical pathway of NF-kappaB. Blood 113, 2755-2764. 


\section{Figure legends}

Figure 1. Spliced and unspliced $H B Z$ genes.

Schema of the spliced and unsplicd $H B Z$ genes is shown (A). Structure of tax gene is also shown. The coding sequences of the $H B Z$ and tax gene do not overlap. The $\mathrm{N}$-terminal region of predicted amino acid sequences are shown in (B).

Figure 2. Three mechanisms to inactivate Tax expression in ATL cells

1) genetic changes of the tax gene (nonsense mutation, deletion and insertion); 2) DNA methylation of 5'LTR silences transcription of the tax gene; and 3) deletion of 5'LTR, which is the promoter/enhancer of the tax gene transcription.

Figure 3. Functional domains of HBZ

HBZ has three domains: activation, central and bZIP. Functions of each domain are summarized.

Figure 4. Functions of HBZ

HBZ protein interacts with c-Jun or CREB and suppresses viral transcription from the 5'LTR. HBZ mRNA promotes T-cell proliferation. A transcription factor, SP1, plays a critical role in the $H B Z$ gene transcription. Detailed description can be found in the text. 

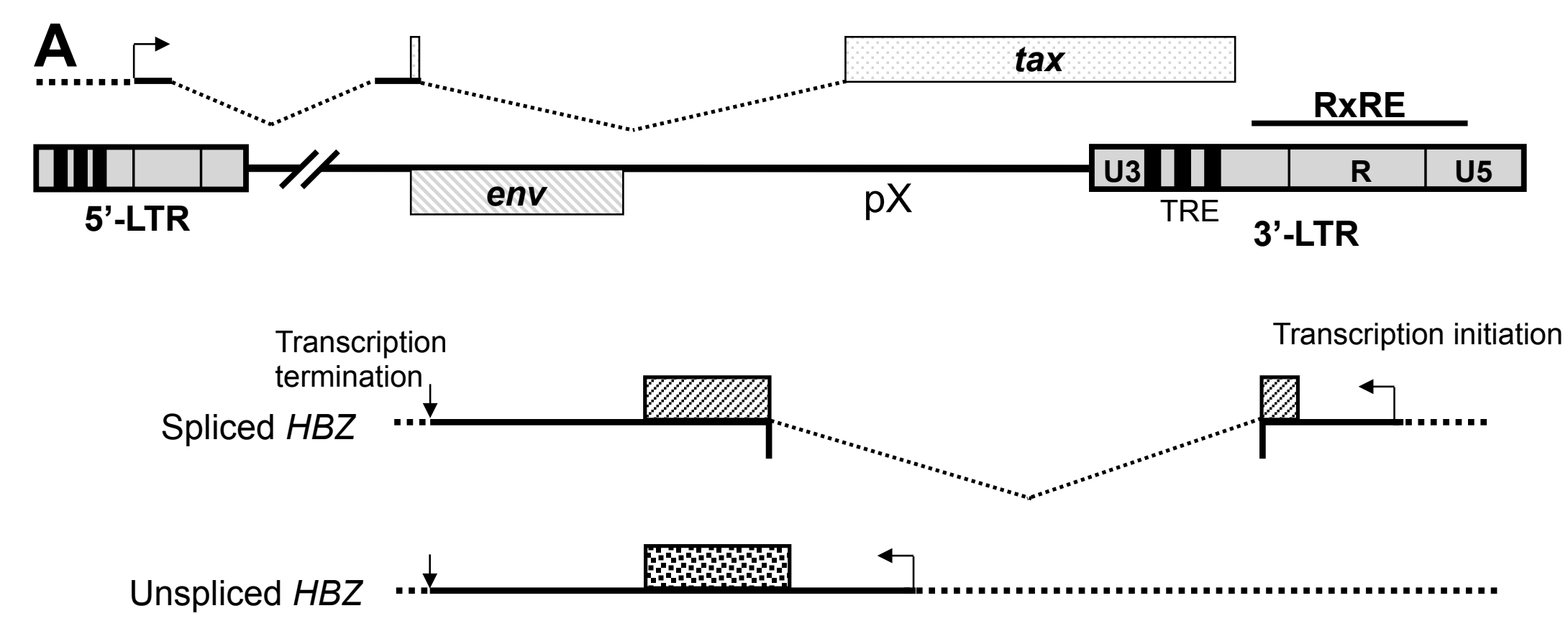

B

$\begin{aligned} \text { Spliced HBZ } & \text {---MAASGLFRCLPVSCPED------ } \\ \text { Unspliced HBZ } & \text { MVNFVSAGLFRCLPVSCPED------ }\end{aligned}$



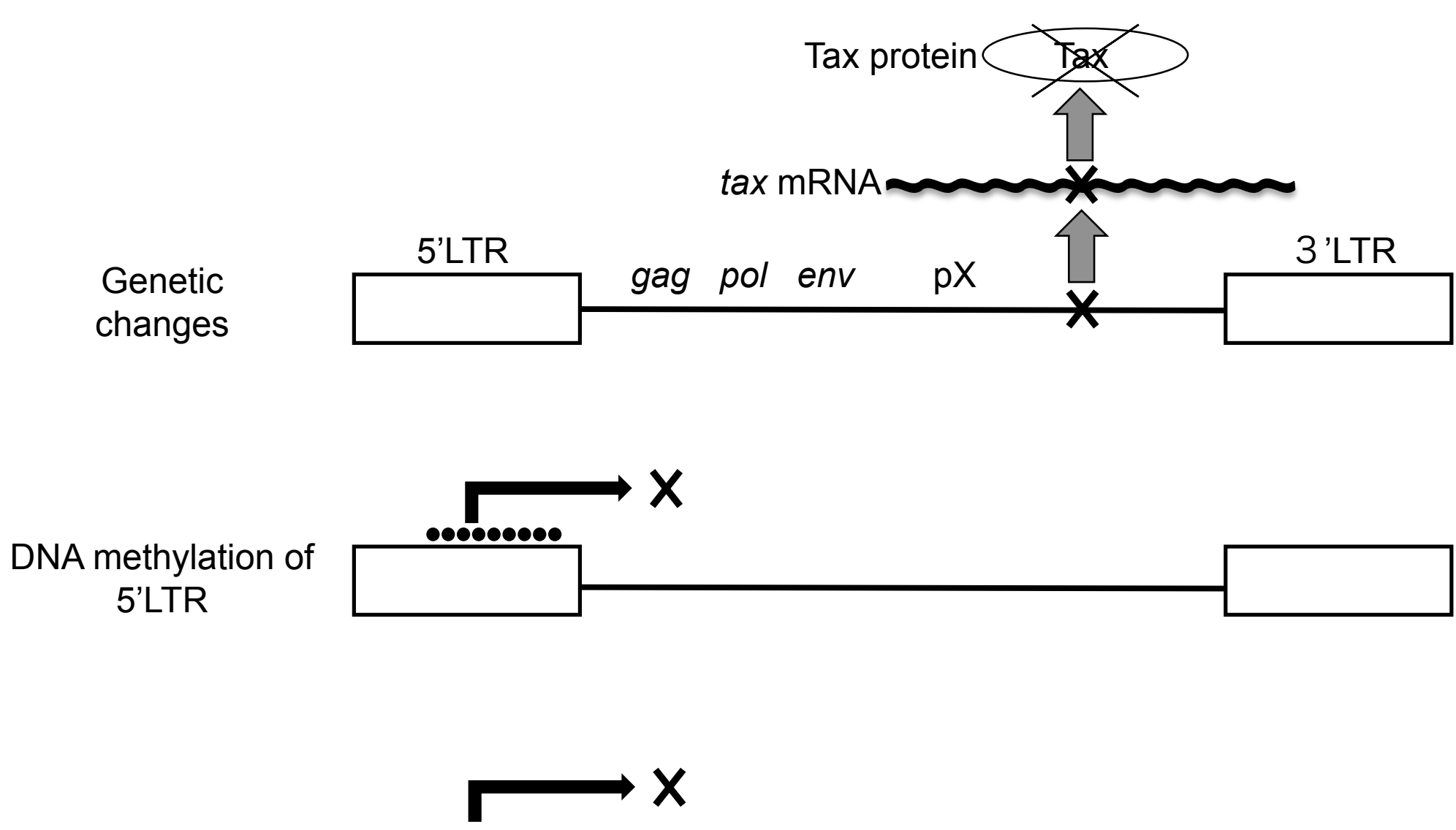

Deletion of 5'LTR

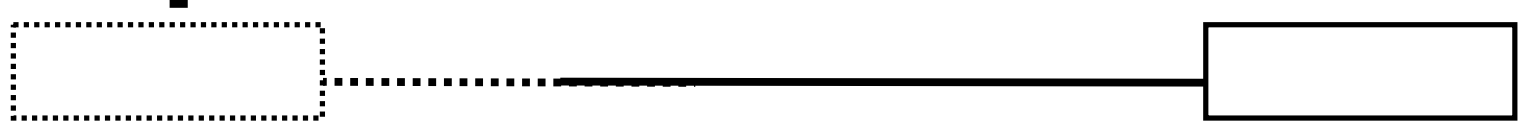




\section{AD $\quad C D \quad b Z I P$}

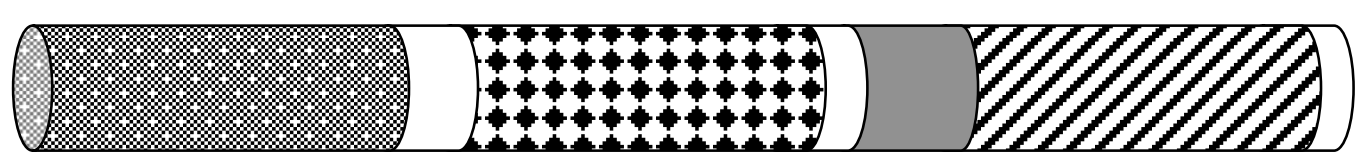

AD: activation domain

$C D$ : central domain

bZIP: basic ZIP domain

\begin{tabular}{|c|l|}
\hline AD & $\begin{array}{l}\text { *Binding with 26S proteasome (usHBZ) } \\
\text { Degradation of c-Jun } \\
\text { *Interaction with p300 }\end{array}$ \\
\hline CD & *Nuclear localization \\
\hline AD+bZIP & $\begin{array}{l}\text { *Binding with p65, inhibition of classical NF-kB pathway } \\
\text { *Increase of hTERT promoter activity } \\
\text { *Activation of JunD }\end{array}$ \\
\hline bZIP & *Interaction and inhibition of c-Jun, Jun B, CREB, CREB2 \\
\hline
\end{tabular}




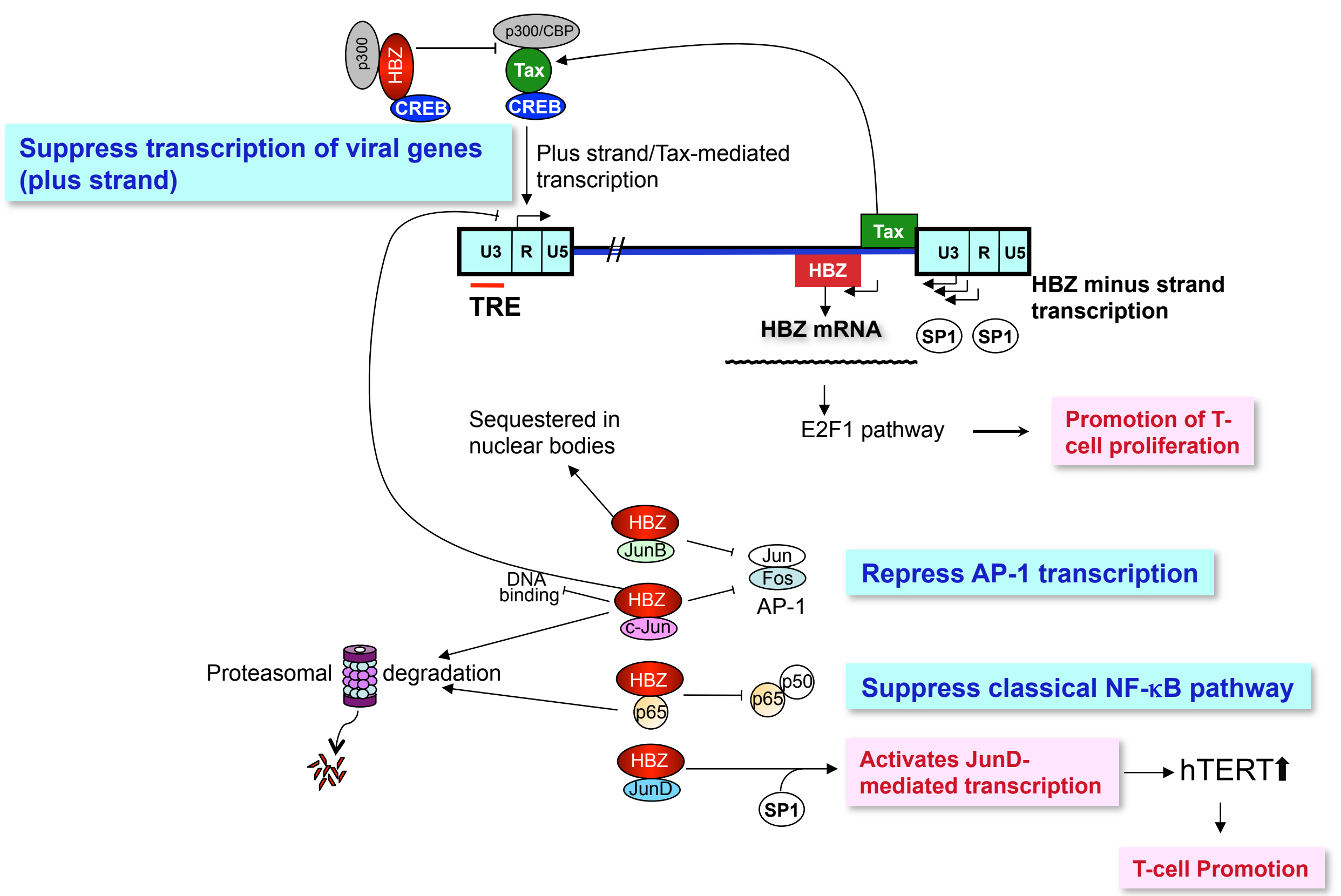

